\title{
Appendix 5 \\ Tenant registry
}

\section{Csengery Street 64}

\begin{tabular}{|c|c|c|c|c|c|c|c|}
\hline & 1941 & $\begin{array}{l}\text { Occu- } \\
\text { pation }\end{array}$ & $\begin{array}{l}\text { Moved } \\
\text { in }\end{array}$ & $\begin{array}{l}\text { Reconstruction of } \\
\text { October } 15,1944 \\
\text { state of affairs }\end{array}$ & 1945 & $\begin{array}{l}\text { Occu- } \\
\text { pation }\end{array}$ & $\begin{array}{l}\text { Moved } \\
\text { in }\end{array}$ \\
\hline \multicolumn{8}{|l|}{ GF } \\
\hline 1 & $\begin{array}{l}\text { József } \\
\text { Strucky } 1895 \\
\text { Mrs. Strucky } \\
1901 \\
\text { Éva Strucky } \\
1926\end{array}$ & file cutter & 1929 & $\begin{array}{l}\text { József Strucky } \\
\text { Mrs. Strucky } \\
\text { Éva Strucky } \\
\text { Lodger: } \\
\text { György Széplaki } \\
\text { (Szedlacsek) }\end{array}$ & $\begin{array}{l}\text { János Pál } 1907 \\
\text { Mrs. Pál } 1915 \\
\text { Erzsébet Pál } 1934 \\
\text { Éva Pál } 1940 \\
\text { Co-tenants: } \\
\text { Mihály Sáska } \\
1912 \\
\text { Mrs. Sáska } 1918 \\
\text { László } \\
\text { Sáska } 1941\end{array}$ & $\begin{array}{l}\text { file cutter } \\
\text { under- } \\
\text { worker } \\
\text { employed } \\
\text { at the } \\
\text { Beszkárt }\end{array}$ & $\begin{array}{l}1944 \\
\text { December } \\
1945 \\
\text { January }\end{array}$ \\
\hline 2 & $\begin{array}{l}\text { Mrs. Izsák } \\
\text { Grünberger } \\
\text { née Sári } \\
\text { Müller } 1903\end{array}$ & trader & 1937 & $\begin{array}{l}\text { Ernő Grünberger } \\
\text { Mrs. István Várnai } \\
\text { Izsák Grünberger } \\
\text { Mrs. Izsák Grün- } \\
\text { berger } \\
\text { Mrs. Grossmann née } \\
\text { Olga Grünberger } \\
\text { Ilona Grünberger } \\
\text { Margit Grünberger } \\
\text { Vilmos Grünberger }\end{array}$ & $\begin{array}{l}\text { Ernő Grünberger } \\
1903 \\
\text { Mrs. István Várnai } \\
1896\end{array}$ & & 1944 \\
\hline $2 a$ & $\begin{array}{l}\text { Alfréd } \\
\text { Köszler } 1893 \\
\text { Mrs. Köszler } \\
1891 \\
\text { Their child } \\
1927\end{array}$ & pressman & 1937 & $\begin{array}{l}\text { Mrs. Miklós Rosen- } \\
\text { berg } \\
\text { Magda Rosenberg } \\
\text { Edit } \\
\text { Rosenberg }\end{array}$ & $\begin{array}{l}\text { Miklós } \\
\text { Rosenberg } 1905 \\
\text { Mrs. Rosenberg } \\
1929 \\
\text { Magda } \\
\text { Rosenberg } 1929 \\
\text { Edit } \\
\text { Rosenberg } 1931\end{array}$ & tinker & $\begin{array}{l}1942 \\
\text { September }\end{array}$ \\
\hline
\end{tabular}

Note: Reconstruction based on the court testimonies and the interviews. The victims' names are with bold. 
Continued

\begin{tabular}{|c|c|c|c|c|c|c|c|}
\hline & 1941 & $\begin{array}{l}\text { Occu- } \\
\text { pation }\end{array}$ & $\begin{array}{l}\text { Moved } \\
\text { in }\end{array}$ & $\begin{array}{l}\text { Reconstruction of } \\
\text { October } 15,1944 \\
\text { state of affairs }\end{array}$ & 1945 & $\begin{array}{l}\text { Occu- } \\
\text { pation }\end{array}$ & $\begin{array}{l}\text { Moved } \\
\text { in }\end{array}$ \\
\hline \multicolumn{8}{|c|}{ GF } \\
\hline 3 & $\begin{array}{l}\text { Jakab } \\
\text { Schwartz } \\
1883 \\
\text { Mrs. Jakab } \\
\text { Schwartz } \\
1894 \\
\text { László } \\
\text { Schwartz } \\
1921\end{array}$ & turner & 1934 & $\begin{array}{l}\text { Mrs. Jakab Schwartz } \\
\text { László } \\
\text { Schwartz } \\
\text { Mrs. Miksa Eisen- } \\
\text { stadter née Elza } \\
\text { Schwartz } \\
\text { Miksa Eisenstadter }\end{array}$ & $\begin{array}{l}\text { Mrs. Jakab } \\
\text { Schwartz } 1894 \\
\text { László Schwartz } \\
1921\end{array}$ & & \\
\hline 4 & $\begin{array}{l}\text { Miksa Licht- } \\
\text { man } 1892 \\
\text { Mrs. Miksa } \\
\text { Lichtman } \\
1891 \\
\text { Mihály Licht- } \\
\text { man } 1930\end{array}$ & $\begin{array}{l}\text { stock } \\
\text { trader }\end{array}$ & 1937 & $\begin{array}{l}\text { Mrs. Miksa Lichtman } \\
\text { Mihály Lichtman }\end{array}$ & $\begin{array}{l}\text { Mrs. Miksa Licht- } \\
\text { man } 1891 \\
\text { Mihály Lichtman } \\
1910 \\
\text { Co-tenant: Mrs. } \\
\text { Adolf Pálmai } 1897\end{array}$ & 1945 & \\
\hline 5 & $\begin{array}{l}\text { Géza Hámori } \\
1905 \\
\text { Mrs. Hámori } \\
1907 \\
\text { Éva Hámori } \\
1932 \\
\text { Béla Hámori } \\
1937\end{array}$ & $\begin{array}{l}\text { post } \\
\text { officer }\end{array}$ & & & $\begin{array}{l}\text { Mrs. Ármin Lich- } \\
\text { tenstein } 1895 \\
\text { Olga Gradt } 1916 \\
\text { Györgyi Hade } \\
1940 \\
\text { Co-tenants: Mrs. } \\
\text { Weisz 1899 } \\
\text { Rózsi Weisz } 1831\end{array}$ & & June 1944 \\
\hline & $\begin{array}{l}\text { Ede Hartrick } \\
1893 \\
\text { Mrs. Ede } \\
\text { Hartrick } \\
1875\end{array}$ & $\begin{array}{l}\text { vice } \\
\text { janitor }\end{array}$ & 1921 & & $\begin{array}{l}\text { Mrs. Ede Hartrick } \\
1875\end{array}$ & & \\
\hline 8 & $\begin{array}{l}\text { György } \\
\text { Szamocseta } \\
1891 \\
\text { Mrs. György } \\
\text { Szamocseta } \\
1900 \\
\text { Lenke } \\
\text { Szamocseta } \\
1926\end{array}$ & tailor & 1937 & & & & \\
\hline
\end{tabular}


Continued

\begin{tabular}{|c|c|c|c|c|c|c|c|}
\hline & 1941 & $\begin{array}{l}\text { Occu- } \\
\text { pation }\end{array}$ & $\begin{array}{l}\text { Moved } \\
\text { in }\end{array}$ & $\begin{array}{l}\text { Reconstruction of } \\
\text { October } 15,1944 \\
\text { state of affairs }\end{array}$ & 1945 & $\begin{array}{l}\text { Occu- } \\
\text { pation }\end{array}$ & $\begin{array}{l}\text { Moved } \\
\text { in }\end{array}$ \\
\hline \multicolumn{8}{|l|}{ GF } \\
\hline \multicolumn{8}{|c|}{ 1st floor } \\
\hline 1 & $\begin{array}{l}\text { Béla Krámer } \\
\text { Mrs. Béla } \\
\text { Krámer } \\
\text { Magda } \\
\text { Krámer } 1932 \\
\text { Maid: } \\
\text { Erzsébet } \\
\text { Torma } 1893\end{array}$ & butcher & $\begin{array}{l}1938 \\
1941\end{array}$ & $\begin{array}{l}\text { Béla Krámer } \\
\text { Samu Éber } \\
\text { Mrs. Béla Krámer } \\
\text { née Irén Schlesinger } \\
\text { Magda Krámer Gábor } \\
\text { Éber } \\
\text { Mrs. Jenő Éber Bora } \\
\text { Donáth } \\
\text { Ágnes Éber } \\
\text { Mrs. Pál Beck Julia } \\
\text { Schlesinger } \\
\text { Vera Beck } \\
\text { Ernő Blitz } \\
\text { Mrs. Blitz }\end{array}$ & $\begin{array}{l}\text { Béla Krámer } \\
\text { Mrs. Béla Krámer } \\
1910 \\
\text { Ilona Krámer } 1932 \\
\text { Lodger: József } \\
\text { Krámer } 1907\end{array}$ & $\begin{array}{l}\text { home- } \\
\text { maker }\end{array}$ & \\
\hline $1 a$ & $\begin{array}{l}\text { János Jellinek } \\
1870\end{array}$ & physician & 1938 & $\begin{array}{l}\text { János Jellinek } \\
\text { Mrs. János Jellinek } \\
\text { Kamillo Jellinek }\end{array}$ & $\begin{array}{l}\text { Mrs. János Jellinek } \\
1881 \\
\text { Lodgers: Mrs. } \\
\text { Miksa Bodor } 1892 \\
\text { Mrs. Jakab Stein } \\
1964\end{array}$ & $\begin{array}{l}\text { home- } \\
\text { maker }\end{array}$ & 1945 \\
\hline 2 & $\begin{array}{l}\text { Mrs. Henrik } \\
\text { Bodor } 1869 \\
\text { Maid: Katalin } \\
\text { Révész }\end{array}$ & $\begin{array}{l}\text { house } \\
\text { owner }\end{array}$ & 1940 & & $\begin{array}{l}\text { Ilona Bodor } \\
\text { Lodgers: } \\
\text { Miklós Bodor } \\
1932 \\
\text { László Bodor } 1928 \\
\text { Ármin Leindhofer } \\
1895 \\
\text { Mrs. Ármin } \\
\text { Leindhofer } 1895 \\
\text { András Leindhofer } \\
1916 \\
\text { Tamás Leindhofer } \\
1942\end{array}$ & & 1945 \\
\hline $2 a$ & $\begin{array}{l}\text { Asztrik Braun } \\
1892 \\
\text { Mrs. Asztrik } \\
\text { Braun } 1920\end{array}$ & $\begin{array}{l}\text { oil mer- } \\
\text { chant }\end{array}$ & 1938 & $\begin{array}{l}\text { Remembered as Elza } \\
\text { Braun but actually } \\
\text { Erzsébet; probably } \\
\text { Mrs. Rezső Braun } \\
\text { and her two sons }\end{array}$ & $\begin{array}{l}\text { Pál Hegyi } 1899 \\
\text { Mrs. Pál Hegyi } \\
1920 \\
\text { Lodgers: } \\
\text { Katalin Kurcweil } \\
1916 \\
\text { László Kurcweil } \\
1932\end{array}$ & $\begin{array}{l}\text { retired } \\
\text { auditor }\end{array}$ & $\begin{array}{l}\text { November } \\
1944 \\
\\
\text { January } \\
1945\end{array}$ \\
\hline
\end{tabular}


Continued

\begin{tabular}{|c|c|c|c|c|c|c|c|}
\hline & 1941 & $\begin{array}{l}\text { Occu- } \\
\text { pation }\end{array}$ & $\begin{array}{l}\text { Moved } \\
\text { in }\end{array}$ & $\begin{array}{l}\text { Reconstruction of } \\
\text { October 15, } 1944 \\
\text { state of affairs }\end{array}$ & 1945 & $\begin{array}{l}\text { Occu- } \\
\text { pation }\end{array}$ & $\begin{array}{l}\text { Moved } \\
\text { in }\end{array}$ \\
\hline \multicolumn{8}{|l|}{ GF } \\
\hline 3 & $\begin{array}{l}\text { Izsák Grün- } \\
\text { feld } 1883 \\
\text { Mrs. Izsák } \\
\text { Grünfeld } \\
1892\end{array}$ & jeweller & 1928 & $\begin{array}{l}\text { Izsák Grünfeld } \\
\text { Mrs. Izsák Grünfeld }\end{array}$ & $\begin{array}{l}\text { Károly Fok } 1903 \\
\text { Mrs. Károly Fok } \\
1903 \\
\text { Lodgers: } \\
\text { Károly Fogl } 1895 \\
\text { Mrs. Fogl } 1893\end{array}$ & $\begin{array}{l}\text { Beszkárt } \\
\text { driver } \\
\text { jeweller }\end{array}$ & $\begin{array}{l}\text { December } \\
1944\end{array}$ \\
\hline 4 & $\begin{array}{l}\text { Ernő Spitzer } \\
1901 \\
\text { Miksa Spitzer } \\
1872\end{array}$ & $\begin{array}{l}\text { central } \\
\text { heating } \\
\text { techni- } \\
\text { cian }\end{array}$ & 1936 & $\begin{array}{l}\text { Ernő Spitzer (Sárdi) } \\
\text { Mrs. Spitzer née } \\
\text { Kató Berkovits } \\
\text { Mrs. Ickovits } \\
\text { Ágnes Ickovits } \\
\text { Tibor Ickovits }\end{array}$ & $\begin{array}{l}\text { Ernő Spitzer } \\
\text { Mrs. Ernő Spitzer } \\
1910\end{array}$ & & \\
\hline 5 & $\begin{array}{l}\text { Mrs. Ignác } \\
\text { Reiner } \\
\text { Ernő (Ödön) } \\
\text { Singer } 1884 \\
\text { Mrs. Zoltán } \\
\text { Kahn née } \\
\text { Róza Stern } \\
1899 \\
\text { István Ehlich } \\
1909\end{array}$ & $\begin{array}{l}\text { trades- } \\
\text { man }\end{array}$ & 1936 & $\begin{array}{l}\text { Ödön Singer } \\
\text { Mrs. Ödön Singer } \\
\text { Her sister-in-law } \\
\text { Ernő Singer }\end{array}$ & $\begin{array}{l}\text { Mrs. Ignác Reiner } \\
\text { Rózsa Singer } 1874 \\
\text { Co-tenant: } \\
\text { Ödön Singer } 1886 \\
\text { Ödön Singer } 1887 \\
\text { Lodger: Sándor } \\
\text { Sebők } 1909\end{array}$ & & 1945 \\
\hline 6 & $\begin{array}{l}\text { Ervin Gábor } \\
1903\end{array}$ & $\begin{array}{l}\text { trades- } \\
\text { man }\end{array}$ & 1939 & $\begin{array}{l}\text { Izidor (Izrael) Lichter } \\
\text { Mrs. Izidor Lichter } \\
\text { Mrs. Andor Lichter } \\
\text { née Halász Irén } \\
\text { György Lichter } \\
\text { Mrs. Ervin Gábor } \\
\text { née Magda Lichter } \\
\text { Zsuzsa Gábor } \\
\text { Mrs. Bierman }\end{array}$ & $\begin{array}{l}\text { Mrs. Ervin Gábor } \\
\text { née Magda Lichter } \\
1908 \\
\text { Zsuzsa Gábor } \\
1940 \\
\text { Mrs. Izrael Lichter } \\
1874 \\
\text { Andor Lichter } \\
1890\end{array}$ & $\begin{array}{l}\text { clerk at a } \\
\text { publishing } \\
\text { house }\end{array}$ & \\
\hline \multicolumn{8}{|c|}{$2^{\text {nd }}$ floor } \\
\hline 1 & $\begin{array}{l}\text { Mrs. Mózes } \\
\text { Rubinyi née } \\
\text { Ilona Bodor } \\
1924 \\
\text { Mrs. György } \\
\text { Rejtő } 1915\end{array}$ & & 1924 & $\begin{array}{l}\text { Mrs. Mózes Rubinyi } \\
\text { née Ilona Bodor }\end{array}$ & $\begin{array}{l}\text { Pál Gráber } 1898 \\
\text { Mrs. Pál Gráber } \\
1904 \\
\text { Hedvig Gráber } \\
1925 \\
\text { Mária Gráber } \\
1932 \\
\text { Co-tenant: Mrs. } \\
\text { Mór Rajna } 1875\end{array}$ & auditor & 1945 \\
\hline 2 & $\begin{array}{l}\text { István Faragó } \\
1898 \\
\text { Mrs. Faragó } \\
\text { née Júlia } \\
\text { Frankl } 1906\end{array}$ & $\begin{array}{l}\text { bank } \\
\text { clerk, } \\
\text { Hungari- } \\
\text { an Credit } \\
\text { Bank }\end{array}$ & 1939 & $\begin{array}{l}\text { István Faragó } \\
\text { Mrs. István Faragó } \\
\text { Lajos (István) Faragó } \\
\text { lodger }\end{array}$ & $\begin{array}{l}\text { Imre Frankl } 1907 \\
\text { Lodger: } \\
\text { Mrs. Solf }\end{array}$ & lawyer & \\
\hline
\end{tabular}


Continued

\begin{tabular}{|c|c|c|c|c|c|c|c|}
\hline & 1941 & $\begin{array}{l}\text { Occu- } \\
\text { pation }\end{array}$ & $\begin{array}{l}\text { Moved } \\
\text { in }\end{array}$ & $\begin{array}{l}\text { Reconstruction of } \\
\text { October } 15,1944 \\
\text { state of affairs }\end{array}$ & 1945 & $\begin{array}{l}\text { Occu- } \\
\text { pation }\end{array}$ & $\begin{array}{l}\text { Moved } \\
\text { in }\end{array}$ \\
\hline \multicolumn{8}{|c|}{ GF } \\
\hline 3 & $\begin{array}{l}\text { Béla Bodor } \\
1895 \\
\text { Mrs. Béla } \\
\text { Bodor } 1904 \\
\text { László Bodor } \\
1928 \\
\text { Miklós Bodor } \\
1932 \\
\text { Katalin Bodor } \\
1914\end{array}$ & $\begin{array}{l}\text { Hungari- } \\
\text { an bank } \\
\text { clerk }\end{array}$ & 1929 & $\begin{array}{l}\text { Béla Bodor } \\
\text { Mrs. Béla Bodor } \\
\text { László Bodor } \\
\text { Miklós Bodor } \\
\text { Katalin Bodor }\end{array}$ & $\begin{array}{l}\text { Béla Czóbel } 1904 \\
\text { Mrs. Béla Czóbel } \\
1908 \\
\text { Mária Czóbel } 1930 \\
\text { Márta Czóbel } \\
1930 \\
\text { Margit Czóbel } \\
1933 \\
\text { Béla Czóbel } 1935 \\
\text { Emília Czóbel } \\
1937 \\
\text { Gábor Czóbel } \\
\text { 1939 } \\
\text { Co-tenant: the } \\
\text { maid }\end{array}$ & $\begin{array}{l}\text { municipal } \\
\text { clerk }\end{array}$ & $\begin{array}{l}\text { December } \\
1944\end{array}$ \\
\hline 4 & $\begin{array}{l}\text { Ernő Léderer } \\
1882 \\
\text { István Léde- } \\
\text { rer } 1912 \\
\text { Lodgers: } \\
\text { Anna Bitter } \\
1913 \\
\text { Rózsa Bitter } \\
1909\end{array}$ & $\begin{array}{l}\text { textile } \\
\text { trader }\end{array}$ & 1912 & $\begin{array}{l}\text { Mrs. Ernő Léderer } \\
\text { née Gizella Scheffer }\end{array}$ & $\begin{array}{l}\text { Mrs. Ernő Léderer } \\
\text { née Gizella } \\
\text { Scheffer } 1888 \\
\text { Lodgers: } \\
\text { Mrs. Ernő Török } \\
1893 \\
\text { Emma Léderer } \\
1888\end{array}$ & $\begin{array}{l}\text { home- } \\
\text { maker }\end{array}$ & \\
\hline 5 & $\begin{array}{l}\text { Zsuzsa Stein- } \\
\text { er } 1918 \\
\text { Endre Steiner } \\
1920\end{array}$ & $\begin{array}{l}\text { textile } \\
\text { trader } \\
\text { employee }\end{array}$ & 1936 & $\begin{array}{l}\text { Mrs. Ármin Steiner } \\
\text { Mrs. Somogyi née } \\
\text { Zsuzsa Steiner } \\
\text { Dezső Somogyi } \\
\text { Andor (Endre) Stein- } \\
\text { er } \\
\text { Mrs. Andor Steiner } \\
\text { István Steiner } \\
\text { Lajos Steiner } \\
\text { Mrs. Lajos Steiner } \\
\text { née Olga Mitzaki } \\
\text { Béla Steiner } \\
\text { Mrs. Miksa Tenczer } \\
\text { Tenczer Róbert } \\
\text { Ernő Singer } \\
\text { Two unknown men: } \\
\text { Faragó, Faragó }\end{array}$ & $\begin{array}{l}\text { Mrs. Ármin Steiner } \\
\text { née Ilona Fürst } \\
1932 \\
\text { Mrs. Dávid Pred- } \\
\text { berger } 1915 \\
\text { András Predberger } \\
1943\end{array}$ & nurse & \\
\hline
\end{tabular}


Continued

\begin{tabular}{|c|c|c|c|c|c|c|c|}
\hline & 1941 & $\begin{array}{l}\text { Occu- } \\
\text { pation }\end{array}$ & $\begin{array}{l}\text { Moved } \\
\text { in }\end{array}$ & $\begin{array}{l}\text { Reconstruction of } \\
\text { October } 15,1944 \\
\text { state of affairs }\end{array}$ & 1945 & $\begin{array}{l}\text { Occu- } \\
\text { pation }\end{array}$ & $\begin{array}{l}\text { Moved } \\
\text { in }\end{array}$ \\
\hline \multicolumn{8}{|l|}{ GF } \\
\hline 6 & $\begin{array}{l}\text { Zsigmond } \\
\text { Kanitz } 1861\end{array}$ & clerk & 1935 & & $\begin{array}{l}\text { Mrs. Zsigmond } \\
\text { Kanitz née Erzsé- } \\
\text { bet Haas } 1870 \\
\text { Co-tenants: } \\
\text { Albert Dunai } 1914 \\
\text { Mrs. Albert Dunai } \\
1914 \\
\text { Albert Dunai Jr } \\
1941\end{array}$ & $\begin{array}{l}\text { home- } \\
\text { maker }\end{array}$ & \\
\hline 7 & $\begin{array}{l}\text { Ignác Stei- } \\
\text { nicz } 1897 \\
\text { Mrs. Ignác } \\
\text { Steinicz née } \\
\text { Paula Neu- } \\
\text { feld } 1884 \\
\text { Endre Szász } \\
1898 \\
\text { Mrs. Szász } \\
\text { née Katalin } \\
\text { Steincz } 1914\end{array}$ & $\begin{array}{l}\text { shopclerk } \\
\text { clerk }\end{array}$ & $\begin{array}{l}1934 \\
1939\end{array}$ & $\begin{array}{l}\text { Károly Réti } \\
\text { Mrs. Károly Réti her } \\
\text { two sisters and a } \\
\text { nephew }\end{array}$ & $\begin{array}{l}\text { András Marosvöl- } \\
\text { gyi } 1901 \\
\text { Mrs. Marosvölgyi } \\
1907 \\
\text { Erzsébet Maros- } \\
\text { völgyi } 1918 \\
\text { Piroska Marosvöl- } \\
\text { gyi } 1931 \\
\text { Co-tenant: } \\
\text { Kálmán Szalay } \\
1903 \\
\text { Mrs. Szalay } 1904 \\
\text { Mrs. András } \\
\text { Marcsa } 1878\end{array}$ & handyman & $\begin{array}{l}\text { December } \\
1944\end{array}$ \\
\hline \multicolumn{8}{|c|}{$3^{\text {rd }}$ floor } \\
\hline 1 & $\begin{array}{l}\text { Mrs. Ignác } \\
\text { Stern } 1884 \\
\text { Lodgers: } \\
\text { Irén Rédecsi } \\
1920 \\
\text { Karl Kurt } \\
\text { Shobez } 1913\end{array}$ & $\begin{array}{l}\text { coopera- } \\
\text { tive soci- } \\
\text { ety clerk }\end{array}$ & 1920 & $\begin{array}{l}\text { Ignác Stern } \\
\text { Mrs. Ignác Stern }\end{array}$ & $\begin{array}{l}\text { Endre István Tóth } \\
\text { MD } 1919\end{array}$ & physician & $\begin{array}{l}\text { December } \\
1944\end{array}$ \\
\hline 2 & $\begin{array}{l}\text { Gyula Por- } \\
\text { derák } 1899 \\
\text { Mrs. Porde- } \\
\text { rák née } \\
\text { Sarolta Bos- } \\
\text { sényi } 1899 \\
\text { Henrik Jáger } \\
1870 \\
\text { Maid: Lídia } \\
\text { Bede } 1920\end{array}$ & $\begin{array}{l}\text { coopera- } \\
\text { tive soci- } \\
\text { ety clerk }\end{array}$ & 1939 & & $\begin{array}{l}\text { József Strucky }{ }^{418} \\
1895 \\
\text { Mrs. József } \\
\text { Strucky } 1901 \\
\text { Éva Strucky } 1926\end{array}$ & & $\begin{array}{l}\text { February } \\
1945\end{array}$ \\
\hline
\end{tabular}

418 The Struckys moved here after Mrs. Krámer had succefully reclaimed her $1^{\text {st }}$ floor apartment. 
Continued

\begin{tabular}{|c|c|c|c|c|c|c|c|}
\hline & 1941 & $\begin{array}{l}\text { Occu- } \\
\text { pation }\end{array}$ & $\begin{array}{l}\text { Moved } \\
\text { in }\end{array}$ & $\begin{array}{l}\text { Reconstruction of } \\
\text { October } 15,1944 \\
\text { state of affairs }\end{array}$ & 1945 & $\begin{array}{l}\text { Occu- } \\
\text { pation }\end{array}$ & $\begin{array}{l}\text { Moved } \\
\text { in }\end{array}$ \\
\hline \multicolumn{8}{|c|}{ GF } \\
\hline 3 & $\begin{array}{l}\text { Gábor } \\
\text { Kardos } 1881 \\
\text { László Kar- } \\
\text { dos } 1918 \\
\text { Maid: } \\
\text { Erzsébet } \\
\text { Nyitári } 1919\end{array}$ & $\begin{array}{l}\text { trader, } \\
\text { salesman }\end{array}$ & 1937 & $\begin{array}{l}\text { Adolf Klein } \\
\text { Mrs. Adolf Klein } \\
\text { Ilona Klein } \\
\text { her sister Babi } \\
\text { László Propper law- } \\
\text { yer } \\
\text { Mrs. László Propper } \\
\text { (Lili) }\end{array}$ & $\begin{array}{l}\text { Mrs. László Kardos } \\
\text { née Éva Szász } \\
1922 \\
\text { Mrs. Pryna } 1903 \\
\text { Mrs. Ernő Rácz } \\
1897 \\
\text { Irén Szász } 1928 \\
\text { Ferenc Braun } 1972 \\
\text { Co-tenants: } \\
\text { Mrs. László Prop- } \\
\text { per 1913 } \\
\text { Ernő Klein } 1886 \\
\text { Mrs. Ernő Klein } \\
\text { 1886 } \\
\text { Lujza Klein } 1910\end{array}$ & $\begin{array}{l}\text { women's } \\
\text { tailor } \\
\text { teacher } \\
\text { milliner }\end{array}$ & 1937 \\
\hline 4 & $\begin{array}{l}\text { Mrs. Pál } \\
\text { Boller } 1895\end{array}$ & dresser & 1929 & & $\begin{array}{l}\text { Mrs. Pál Boller } \\
1894 \\
\text { Co-tenants: } \\
\text { Herbert Kollina } \\
1902 \\
\text { Mrs. Kollina } 1916\end{array}$ & $\begin{array}{l}\text { home- } \\
\text { maker } \\
\text { auditor } \\
\text { clerk }\end{array}$ & $\begin{array}{l}1929 \\
1944\end{array}$ \\
\hline 5 & $\begin{array}{l}\text { Mrs. Andor } \\
\text { Balázs née } \\
\text { Margit } \\
\text { Treitsch } \\
1903 \\
\text { Andor Balázs } \\
1896\end{array}$ & clerk & 1938 & & $\begin{array}{l}\text { Jenő Stern } 1906 \\
\text { Mrs. Jenő Stern } \\
1910 \\
\text { Iván Tibor } 1937 \\
\text { Lodger: } \\
\text { Erzsébet Korn- } \\
\text { hauser } 1915\end{array}$ & technician & 1945 \\
\hline 6 & $\begin{array}{l}\text { Mrs. Sándor } \\
\text { Horschavszky } \\
\text { (Lina) } \\
\text { Her daugh- } \\
\text { ter: Mrs. } \\
\text { Gábor Simor }\end{array}$ & & 1915 & & $\begin{array}{l}\text { Mrs. Gábor Singer } \\
1896 \\
\text { Mrs. Lina Hor- } \\
\text { schavszy } 1867 \\
\text { Mrs. Berkovits } \\
1864 \\
\text { Lodgers: } \\
\text { Mrs. Albert Szabó } \\
\text { 1868 } \\
\text { Mrs. Szegő } 1891\end{array}$ & $\begin{array}{l}\text { clerk } \\
\text { cleaner }\end{array}$ & 1915 \\
\hline
\end{tabular}


Continued

\begin{tabular}{|c|c|c|c|c|c|c|c|}
\hline & 1941 & $\begin{array}{l}\text { Occu- } \\
\text { pation }\end{array}$ & $\begin{array}{l}\text { Moved } \\
\text { in }\end{array}$ & $\begin{array}{l}\text { Reconstruction of } \\
\text { October } 15,1944 \\
\text { state of affairs }\end{array}$ & 1945 & $\begin{array}{l}\text { Occu- } \\
\text { pation }\end{array}$ & $\begin{array}{l}\text { Moved } \\
\text { in }\end{array}$ \\
\hline \multicolumn{8}{|l|}{ GF } \\
\hline 7 & $\begin{array}{l}\text { Miklós } \\
\text { Schneider } \\
1898 \\
\text { Mrs. } \\
\text { Schneider } \\
1897 \\
\text { Mária } \\
\text { Gyekits }\end{array}$ & $\begin{array}{l}\text { women's } \\
\text { tailor }\end{array}$ & 1936 & & $\begin{array}{l}\text { Mrs. Sándor Réti } \\
\text { née Margit Am- } \\
\text { stein } 1902 \\
\text { Róbert László Réti } \\
1927 \\
\text { Erzsébet Amstein } \\
1904 \\
\text { Co-tenants: } \\
\text { Károly Raffman } \\
1906 \\
\text { Mrs. Károly Raff- } \\
\text { man 1905 } \\
\text { András Raffman } \\
1942\end{array}$ & $\begin{array}{l}\text { clerk } \\
\text { book } \\
\text { seller }\end{array}$ & June 1944 \\
\hline $4^{\text {th }}$ & floor & & & & & & \\
\hline 1 & $\begin{array}{l}\text { Zsigmond } \\
\text { Schillinger } \\
1903 \\
\text { Maid: } \\
\text { Krisztina } \\
\text { Nyekő } 1922\end{array}$ & locksmith & 1930 & $\begin{array}{l}\text { Zsigmond Schillinger } \\
\text { Mrs. László Polgár } \\
\text { Lili Schillinger } \\
\text { Endre Polgár } \\
\text { Adolf Kohn, his } \\
\text { mother: Mrs. Kohn, } \\
\text { his brother: Aladár } \\
\text { Kohn }\end{array}$ & & & \\
\hline 2 & $\begin{array}{l}\text { Endre Uprim- } \\
\text { ny } 1903 \\
\text { Mária and } \\
\text { Edit Uprimny } \\
1905 \\
\text { Maid: Anna } \\
\text { Varga } 1920\end{array}$ & $\begin{array}{l}\text { owner of } \\
\text { a ma- } \\
\text { chine } \\
\text { shop }\end{array}$ & 1933 & $\begin{array}{l}\text { Endre Uprimny } \\
\text { Edit Uprimny } \\
\text { Mrs. Imre Kun née } \\
\text { Lili Uprimny } \\
\text { János Kun } \\
\text { Teréz Fischer } \\
\text { Mrs. Geiger } \\
\text { Mrs. Loránt Morton } \\
\text { née Erzsébet Neu- } \\
\text { wirth } \\
\text { Mrs. Neuwrith } \\
\text { Róbert Morton } \\
\text { Éva Morton }\end{array}$ & $\begin{array}{l}\text { Miklós Kis } 1903 \\
\text { Mrs. Miklós Kis } \\
1904 \\
\frac{\text { Co-tenants: János }}{\text { Kun } 1930} \\
\text { Teréz Fischer } 1877\end{array}$ & bank clerk & $\begin{array}{l}\text { June } 22 \text {, } \\
1944\end{array}$ \\
\hline 3 & n. a. & & & $\begin{array}{l}\text { Grandpa Róth } \\
\text { Grandma Róth } \\
\text { Márta Róth and } \\
\text { brother }\end{array}$ & & & \\
\hline 4 & $\begin{array}{l}\text { Miklós } \\
\text { Barabás } \\
1887 \\
\text { Mrs. Barabás } \\
1885\end{array}$ & $\begin{array}{l}\text { assistant } \\
\text { clerk }\end{array}$ & 1935 & $\begin{array}{l}\text { Mrs. Tenczer } \\
\text { Róbert Tenczer }\end{array}$ & $\begin{array}{l}\text { Mrs. Lajos Steiner } \\
\text { née Olga Mitzaki } \\
1892\end{array}$ & $\begin{array}{l}\text { paper- } \\
\text { maker }\end{array}$ & June 1944 \\
\hline
\end{tabular}


Continued

\begin{tabular}{|c|c|c|c|c|c|c|c|}
\hline & 1941 & $\begin{array}{l}\text { Occu- } \\
\text { pation }\end{array}$ & $\begin{array}{l}\text { Moved } \\
\text { in }\end{array}$ & $\begin{array}{l}\text { Reconstruction of } \\
\text { October } 15,1944 \\
\text { state of affairs }\end{array}$ & 1945 & $\begin{array}{l}\text { Occu- } \\
\text { pation }\end{array}$ & $\begin{array}{l}\text { Moved } \\
\text { in }\end{array}$ \\
\hline \multicolumn{8}{|c|}{ GF } \\
\hline \multirow[t]{2}{*}{7} & $\begin{array}{l}\text { Mihály } \\
\text { Nyikolits } \\
1913 \\
\text { Mrs. Nyiko- } \\
\text { lits } 1915\end{array}$ & clerk & 1939 & & $\begin{array}{l}\text { Jenő Spitz } 1884 \\
\text { Mrs. Jenő Spitz } \\
1896 \\
\text { Co-tenant: Mrs. } \\
\text { Ferenc Kertész }\end{array}$ & salesman & June 1944 \\
\hline & storefront & & & & & & \\
\hline 2 & $\begin{array}{l}\text { György } \\
\text { Szamocseta }\end{array}$ & tailor & 1937 & & $\begin{array}{l}\text { György } \\
\text { Szamocseta }\end{array}$ & tailor & 1937 \\
\hline 3 & $\begin{array}{l}\text { Andor } \\
\text { Borson }\end{array}$ & $\begin{array}{l}\text { book- } \\
\text { binder }\end{array}$ & 1910 & & & & \\
\hline 5 & $\begin{array}{l}\text { Vilmos } \\
\text { Kömüves } \\
\text { (Klein) } 1873 \\
\text { Mrs. Klein } \\
1874\end{array}$ & $\begin{array}{l}\text { shoe- } \\
\text { maker }\end{array}$ & 1930 & & $\begin{array}{l}\text { György } \\
\text { Szamocseta }\end{array}$ & tailor & 1937 \\
\hline 6 & $\begin{array}{l}\text { Péter } \\
\text { Bakonyi }\end{array}$ & waiter & 1926 & & Péter Bakonyi & waiter & 1926 \\
\hline 7 & n. a. & & & & Hajdu & & \\
\hline \multirow[t]{5}{*}{8} & József Eisler & chandler & 1908 & & József Eisler & chandler & 1908 \\
\hline & n. a. & & & & $\begin{array}{l}\text { Mrs. Kálmán } \\
\text { Polgár }\end{array}$ & & \\
\hline & n. a. & & & & Ignác Róth & & \\
\hline & n. a. & & & & $\begin{array}{l}\text { Mrs. Miksa } \\
\text { Eisentadter }\end{array}$ & & \\
\hline & n. a. & & & & Iván Ámbori & & \\
\hline
\end{tabular}

